$a=-1$ the $L$ neutrino has maximal helicity and in the case of $a=+1$, non-maximal helicity.

The author is grateful to $M$. Kõiv for many valuable critical remarks.

\title{
REFERENCES
}

1. Sterman, G., Townsend, P. K., Nieuwenhuizen, P., Phys. Rev., D17, № 6, 1501-1517 (1978).

2. Mille r, D. H., SLAC-PUB-2713, Stanford, 1981.

3. Ots, I., Preprint F-9, Tartu, 1979.

Academy of Sciences of the Estonian SSR, Institute of Physics

Received

Apr. 12,1982

EESTI NSV TEADUSTE AKADEEMIA TOIMETISED. 31. KOIDE

FOOSIKA * MATEMAATIKA, 1982, NR. 4

ИЗВЕСТИЯ АКАДЕМИИ НАУК ЭСТОНСКОП ССР. ТОМ 31 ФИЗНКА * МАТЕМАТИКА, 1982, № 4

удК $621.372 .8: 535$

П. АДАМСОН

\section{РАСПРЕДЕЛЕНИЕ ПОТОКА МОЩНОСТИ НАПРАВЛЯЕМЫХ МОД АСИММЕТРИЧНОГО ПЛОСКОГО ДИЭЛЕКТРИЧЕСКОГО воЛноводА}

\author{
P. ADAMSON. TASAPINNALISE ASOMMEETRILISE DIELEKTRILISE LAINEJUHI SUUNATUD \\ MOODIDE VOIMSUSVOO JAOTUS \\ P. ADAMSON. POWER DISTRIBUTION OF THE GUIDED MODES OF AN ASYMMETRIC \\ DIELECTRIC SLAB WAVEGUIDE
}

\section{(Представил К. К. Ребане)}

Плоские диэлектрические волноводы (ПДВ) интенсивно исследуются в настоящее время в связи с широкими возможностями применения их в интегрально-оптических системах передачи и обработки информации $\left[{ }^{1,2}\right]$. Модель ПДВ является основой и для анализа волноводных свойств резонатора полупроводникового гетеролазера $\left[{ }^{3,4}\right]$. Цель данного сообщения - анализ распределения потока мощности в асимметричном ПДВ в зависимости от степени его асимметрии. Представлена также аппроксимационная формула для расчета фактора оптического ограничения (ФОО) ТЕ-мод, показывающая, какая доля полного потока мощности распространяется в среднем слое ПДВ.

Рассмотрим асимметрнчный трехслойный бесконечнопротяженный в двух измерениях ПДВ со скачкообразными изменениями диэлектрических проницаемостей на границах. Степень асимметрии ПДВ характеризуется фактором $\eta=\left(\varepsilon_{1}-\varepsilon_{2}\right)\left(\varepsilon_{1}-\varepsilon_{3}\right)^{-1}$, где $\varepsilon_{1}-$ диэлектрическая проницаемость среднего слоя ПДВ, а $\varepsilon_{2}$ и $\varepsilon_{3}$ - диэлектрические проницаемости крайних слоев $\left(\varepsilon_{1}>\varepsilon_{2,3}, \varepsilon_{3} \geqslant \varepsilon_{2}\right)$. В дальнейшем индекс 1 проставляется у всех величин, характеризующих средний слой с $\varepsilon_{1}$, а индексы 2,3 - у величин, относящихся к крайним слоям с $\varepsilon_{2}$ и $\varepsilon_{3}$ соответственно.

Распределение потока мощности моды ПДВ определяется ФОО моды $\Gamma_{1}$ и факторами делокализации моды $\Gamma_{2,3} \rightarrow$ относительные доли 
потоков мощности в крайних слоях. Выражения для данных величин имеют следующий вид $\left[{ }^{5}\right]$ :

$$
\begin{aligned}
& \Gamma_{1 \sigma}^{(N)}=\left(d+\left(\gamma_{2 \sigma}^{(N)}\right)^{2} \eta^{-1} L_{2 \sigma}^{(N)}+\left(\gamma_{3 \sigma}^{(N)}\right)^{2} L_{3 \sigma}^{(N)}\right)\left(L_{\sigma}^{(N)}\right)^{-1}, \\
& \Gamma_{2 \sigma}^{(N)}=\left(k_{\sigma}^{(N)}\right)^{2} L_{2 \sigma}^{(N)}\left(L_{\sigma}^{(N)} \eta\right)^{-1}, \\
& \Gamma_{3 \sigma}^{(N)}=\left(k_{\sigma}^{(N)}\right)^{2} L_{3 \sigma}^{(N)}\left(L_{\sigma}^{(N)}\right)^{-1},
\end{aligned}
$$

где $k_{\sigma}^{(N)}$ - поперечный волновой вектор в среднем слое ПДВ, выраженный в безразмерных единицах $2 \pi\left(\varepsilon_{1}-\varepsilon_{3}\right)^{1 / 2}\left(\lambda_{0}\right)^{-1}\left(\lambda_{0}-\right.$ длина волны в вакууме), $\gamma_{2 \sigma, 3 \sigma}^{(N)}$ - модули поперечных волновых векторов в крайних слоях в тех же единицах, $d$ - толщина волновода в единицах $(2 \pi)^{-1}\left(\varepsilon_{1}-\varepsilon_{3}\right)^{-1 / 2} \lambda_{0} \quad$ (т. н. приведенная толщина). Индекс $\sigma$ характеризует поляризацию моды (ТЕ- или ТМ-моды), $N$ - номер моды. $L_{\sigma}^{(N)}$ - эффективная толщина ПДВ $\left[{ }^{2,5}\right] . \quad L_{\sigma}^{(N)}=d+L_{2 \sigma}^{(N)}+L_{3 \sigma}^{(N)}$, где $L_{2 \sigma, 3 \sigma}^{(N)}-$ глубина проникновения поля в соответствующий крайний слой. $\cdot L_{2 \mathrm{TE}, 3 \mathrm{TE}}^{(N)}=\left(\stackrel{(N)}{\gamma_{2 \mathrm{TE}, 3 \mathrm{TE}}}\right)^{-1} \quad$ и $\quad L_{2 \mathrm{TM}, 3 \mathrm{TM}}^{(N)}=\left(q_{2 \mathrm{TM}, 3 \mathrm{TM}}^{(N)} \stackrel{(N)}{\gamma_{2} \mathrm{TM}, 3 \mathrm{TM}}\right)^{-1}, \quad$ где $q_{2 \mathrm{TM}, 3 \mathrm{TM}}^{(N)}-$ редукционный фактор $\left[{ }^{2,5}\right] . \Gamma_{1 \sigma}^{(N)}+\Gamma_{2 \sigma}^{(N)}+\Gamma_{3 \sigma}^{(N)}=1$.

Для анализа зависимости величин $\quad \Gamma_{1 \sigma}^{(N)}, \Gamma_{2 \sigma}^{(N)}$ и $\Gamma_{3 \sigma}^{(N)}$ от $\eta$ нами рассчитаны их производные по $\eta$. Для $\frac{\partial \Gamma_{2 \sigma}^{(N)}}{\partial \eta}$ и $\frac{\partial \Gamma_{3 \sigma}^{(N)}}{\partial \eta}$ полу: чены следующие выражения:

$$
\begin{aligned}
\frac{\partial \Gamma_{2 \sigma}^{(N)}}{\partial \eta} & =-\left(\frac{k_{\sigma}^{(N)}}{L_{\sigma}^{(N)} \eta}\right)^{2}\left[L_{2 \sigma}^{(N)}\left(d+L_{3 \sigma}^{(N)}\right)+\right. \\
& \left.+\eta L_{2 \sigma}^{(N)} \frac{\partial L_{3 \sigma}^{(N)}}{\partial \eta}-\eta\left(d+L_{3 \sigma}^{(N)}\right) \frac{\partial L_{2 \sigma}^{(N)}}{\partial \eta}\right], \\
\frac{\partial \Gamma_{3 \sigma}^{(N)}}{\partial \eta} & =\left(\frac{k_{\sigma}^{(N)}}{L_{\sigma}^{(N)}}\right)^{2}\left[\frac{L_{2 \sigma}^{(N)} L_{3 \sigma}^{(N)}}{\eta}+\left(d+L_{2 \sigma}^{(N)}\right) \frac{\partial L_{3 \sigma}^{(N)}}{\partial \eta}-L_{3 \sigma}^{(N)} \frac{\partial L_{2 \sigma}^{(N)}}{\partial \eta}\right],
\end{aligned}
$$

где

$$
\begin{aligned}
& \frac{\partial L_{2 \sigma}^{(N)}}{\partial \eta}=-\left[\frac{\left(L_{2 \sigma}^{(N)}\right)^{3}}{2}\left(1-\Gamma_{2 \sigma}^{(N)}\right)+S_{2 \sigma}^{(N)}\right]<0, \\
& \frac{\partial L_{3 \sigma}^{(N)}}{\partial \eta}=\frac{\left(L_{3 \sigma}^{(N)}\right)^{3}}{2} \Gamma_{2 \sigma}^{(N)}-+S_{3 \sigma}^{(N)}>0
\end{aligned}
$$

и $S_{2 \mathrm{TE}, 3 \mathrm{TE}}^{(N)}=0, \quad S_{2 \mathrm{TM}}^{(N)}=\alpha_{3}\left(1+\varepsilon_{1} \varepsilon_{2}^{-1}\right)\left(L_{3 \mathrm{TM}}^{(N)}+d\right) \Gamma_{2 \mathrm{TM}}^{(N)}, \quad S_{3 \mathrm{TM}}^{(N)}=\alpha_{3}\left(1+\varepsilon_{1} \varepsilon_{3}^{-1}\right) X$ $\times \stackrel{(N)}{L}_{3 \mathrm{TM}} \Gamma_{2 \mathrm{TM}}^{(N)}, \quad$ а $\alpha_{3}-$ относительный скачок диэлектрической проницаемости на границе раздела, $\alpha_{3}=\left(\varepsilon_{1}-\varepsilon_{3}\right) \varepsilon_{1}^{-1}$. Формулы показывают, что при любых возможных значениях $\eta(1 \leqslant \eta<\infty)$ $\frac{\partial \Gamma_{2 \sigma}^{(N)}}{\partial \eta}<0$ и $\frac{\partial \Gamma_{3 \sigma}^{(N)}}{\partial \eta}>0$. Таким образом, в асімметричном ПДВ (приведенная толщина фиксирована) с ростом $\eta$ относительная доля потока мощности всех мод всегда строго монотонно увеличивается 
в том крайнем слое, где диэлектрическая проницаемость больше, и всегда строго монотонно уменьшается в другом.

Производную ФОО по $\eta$ можно представить в следующем виде:

$$
\begin{aligned}
\frac{\partial \Gamma_{1 \sigma}^{(N)}}{\partial \eta} & =-\frac{\Gamma_{2 \sigma}^{(N)} L_{3 \sigma}^{(N)}}{2 L_{\sigma}^{(N)}}\left[1+\eta\left(L_{2 \sigma}^{(N)}\right)^{2}\left(1-\Gamma_{2 \sigma}^{(N)}\right)+\left(L_{3 \sigma}^{(N)}\right)^{2} \Gamma_{1 \sigma}^{(N)}-\right. \\
& \left.-\left(\frac{d+L_{3 \sigma}^{(N)}}{L_{3 \sigma}^{(N)}}\right)\left(\frac{2}{\eta}+\left(L_{2 \sigma}^{(N)}\right)^{2}\left(1-\Gamma_{2 \sigma}^{(N)}\right)\right)+F_{\sigma}^{(N)}\right],
\end{aligned}
$$

где $F_{\mathrm{TE}}^{(N)}=0$, а

$$
\begin{aligned}
& F_{\mathrm{TM}}^{(N)}=2 \alpha_{3} \frac{\left(k_{\mathrm{TM}}^{(N)}\right)^{2}}{L_{\mathrm{TM}}^{(N)}}\left[\left(1+\frac{\varepsilon_{1}}{\varepsilon_{3}}\right)\left(d+\frac{(\eta-1)}{\eta} L_{2 \mathrm{TM}}^{(N)}\right)+\right. \\
& \left.+\left(1+\frac{\varepsilon_{1}}{\varepsilon_{2}}\right)\left(\frac{d+L_{3 \mathrm{TM}}^{(N)}}{L_{3 \mathrm{TM}}^{(N)}}\right)\left(\frac{(\eta-1) L_{3 \mathrm{TM}}^{(N)}-d}{\eta^{2}}\right)\right] .
\end{aligned}
$$

Выражение (4) не является монотонной функцией от $\eta$. Можно показать, что при $\eta=1$ величина $\frac{\partial \Gamma_{1 \sigma}^{(N)}}{\partial \eta}>0$, а при больших значениях $\eta-\frac{\partial \Gamma_{1 \sigma}^{(N)}}{\partial \eta}<0$, причем функция $\frac{\partial \Gamma_{1 \sigma}^{(N)}}{\partial \eta}$ имеет только одну нулевую точку. Следовательно, зависимость ФОО от $\eta$ достигает максимума при определенном значении $\eta$, которое больше единицы, т. е. в асимметричном ПДВ піри его заданной приведенной толщине можно получить бо́льшие значения ФОО, чем в симметричном. Хотя численные расчеты это показали $\left[{ }^{6}\right]$, в общем случае данный факт аналитически доказан не был.

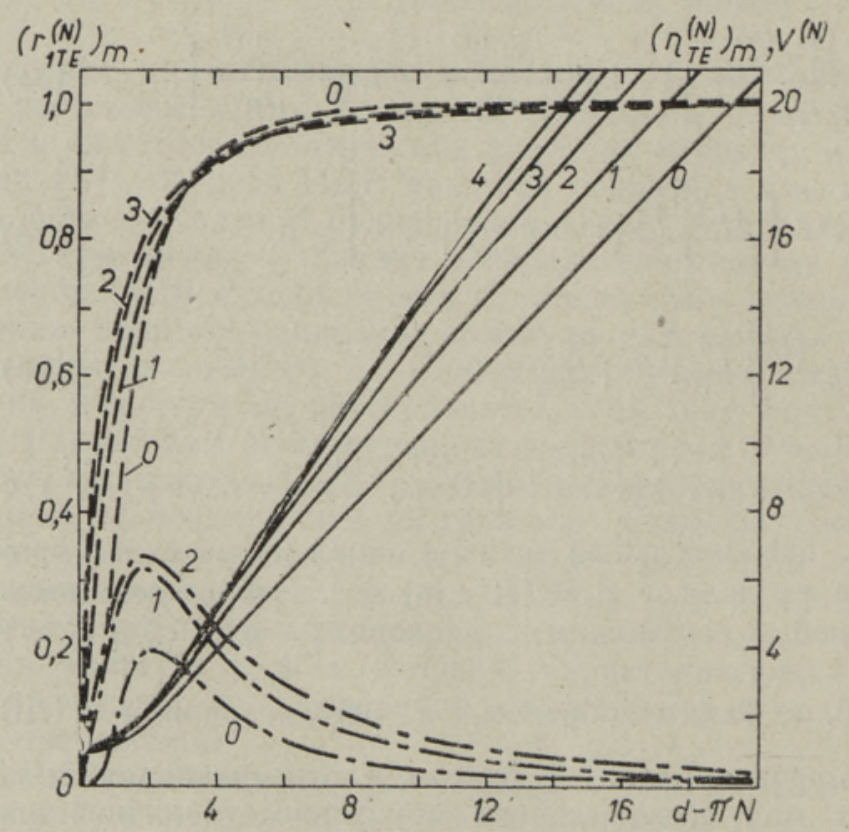

Рис. 1. Зависимость величин $\left(\eta_{\text {те }}^{(N)}\right)_{m} \quad($ сплошные кривые), $\left(\Gamma_{1 \mathrm{TE}}{ }^{(N)}\right)_{m}$ (штриховые кривые) и $V(N) \quad$ (штрих-пунктирные кривые) от приведенной толщины ПДВ.

Цифры у кривых - номера мод (здесь и на рис. 2). 
Уравнение $\frac{\partial \Gamma_{1 \sigma}^{(\hat{N})}}{\partial \eta}=0$ дает значение фактора асимметрии $\left(\eta_{\sigma^{(N)}}\right)_{m}$, при котором ФОО максимален. Аналитическому решению оно не поддается, но легко решается на ЭВМ. Поскольку характеристики ТЕ-мод определяются полностью фактором асимметрии $\eta$ и приведеннсй толщиной $d$, то графики, показывающие зависимость $\left(\eta_{\mathrm{TE}}^{(N)}\right)_{m}$ от $d$, являются универсальными (рис. 1). На рис. 1 показаны также зависимости $\left(\Gamma_{1 \mathrm{TE}}^{(N)}\right)_{m}$ - максимально возможного ФОО асимметричного ПДВ (при факторе асимметрии. $\left.\left(\eta_{\mathrm{TE}}^{(N)}\right)_{m}\right)$ и относительные разности $V^{(N)}=\left(\left(\Gamma_{1 \mathrm{TE}}^{(N)}\right)_{m}-\left(\Gamma_{1 \mathrm{TE}}^{(N)}\right)_{S}\right)\left(\Gamma_{1 \mathrm{TE}}^{(N)}\right)_{S}^{-1}\left(\left(\Gamma_{1 \mathrm{TE}}^{(N)}\right)_{S} \longrightarrow\right.$ ФОО симметричного волновода) в процентах от приведенной толщины $d$ для нескольких низших ТЕ-мод. В случае нулевой ТЕ-моды максимальное увеличение ФОО при переходе от симметричного ПДВ к асимметричному получается при $d \simeq 2$, когда $V^{(0)}$ составляет примерно $4 \%$. С увеличением номера моды $V^{(N)}$ сначала увеличивается, достигая максимального значения у второй моды, затем постепенно уменьшается и при $N=10$ составляет примерно $5 \%$, причем эта зависимость по мере роста $d$ становится более гілоской. В случае ТМ-мод зависимость $\left(\eta_{\mathrm{TM}}^{(N)}\right)_{m}$ от $d$ уже теряет универсальный характер, так как возникает дополнительная зависимость от параметра $\alpha_{3}$.

Поскольку ФОО является важной характеристикой ПДВ, а формула (1) включает и характеристики моды, представляет практический интерес получить аппроксимационную формулу, выражающую ФОО только через параметры асимметрнчного ПДВ, как получена, например, аппроксимационная формула для основной ТЕ-моды симметричного ПДВ $\left[{ }^{7}\right]$. В $\left[{ }^{5}\right]$ для ФОО асимметричных квазипредельных $\quad\left(\gamma_{3 \mathrm{TE}}^{(N)} \ll 1\right)$ TE-мод представлена следующая приближенная формула:

Рис. 2. Зависимость величины $U(N)$ от приведенной толщины ПДВ при четырех факторах асимметрин: $\eta=2 \quad(a-2, \quad$ сплошные кривые); 4 ( $a, 6$, штриховые кривые); $1,5 \quad(6,2$, штриховые кривые); 10 $(a-2$, штрих-пунктирные кривые).
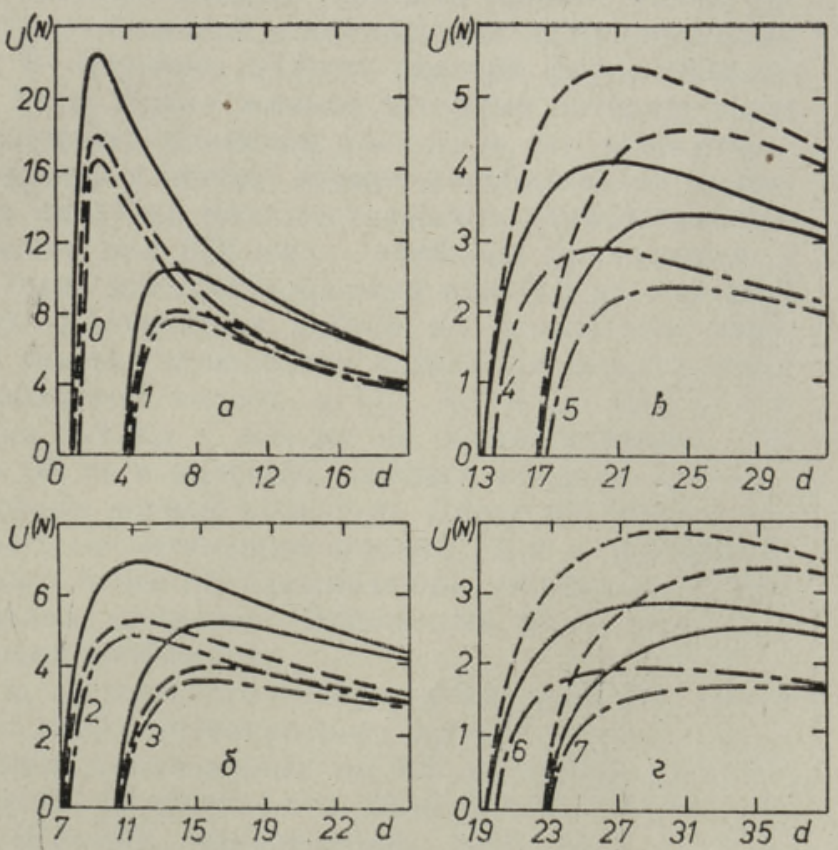


$$
\left(\Gamma_{1 \mathrm{TE}}^{(N)}\right)_{\text {приб } \widetilde{\pi}} \simeq\left[1-L_{2 c}\left(\eta L_{k}\right)^{-1}\right]\left[1-\left(1+t_{a}^{(N)}\right)^{-1 / 2}\right],
$$

где $\quad L_{2 c}=(\eta-1)^{-1 / 2}, \quad L_{k}=d+L_{2 c}, \quad \underset{a}{t_{(N)}}=2 L_{k} \Delta_{a}^{(N)}, \quad \Delta_{a}^{(N)}=d-d_{a}^{(N)} \quad$ и $d_{a}^{(N)}=\pi N+\operatorname{arctg}\left(L_{2 c}^{-1}\right)$. (Физический смысл введенных величин указан в $\left[{ }^{5}\right]$.) Сравнение расчетов ФОО по формулам (6) и (1) показывает, что первая из них достаточно хорошо аппроксимирует ФОО ТЕ-мод асимметричного ПДВ при любых его параметрах. На рис. 2 показаны зависимости относительной ошибки $\quad U^{(N)}=\left(\Gamma_{1 \mathrm{TE}}^{(N)}-\left(\Gamma_{1 \mathrm{TE}}^{(N)}\right)_{\text {прибл }}\right)\left(\Gamma_{1 \mathrm{TE}}^{(N)}\right)^{-1}$ от приведенной толщины при разных значениях фактора асимметрии для первых восьми ТЕ-мод (в процентах). $U^{(N)}$ уменьшается с увеличением номера моды $N$ и фактора асимметрии $\eta$ при данном $N$. Таким образом, аппроксимационную формулу (6) можно успешно применять в случае высших TE-мод сильно асимметричных (один крайний слой воздух) планарных оптических волноводов, представляющих немалый практический интерес [ $\left.{ }^{1}\right]$.

Автор. выражает искреннюю благодарность К. К. Ребане за поддержку работы и В. Г. Федосееву за критические замечания.

\section{ЛИТЕРАТ Р РА}

1. У нгер Х. Г., Планарные и волоконные оптические волноводы, М., «Мир», 1980.

2. Интегральная оптика, М., «Мир», 1978.

3. Богданкевич О. В., Д а рзнек С. А.; Елисеев П. Г., Полупроводниковые лазеры, М., «Наука», 1976.

4. К ей с и Х., Пан иш М., Лазеры на гетероструктурах. т. 1. Основные принципы, М., «Мир», 1981.

5. Фед осеев В. Г., Ад а мсон П. В., Квант. электроника, 9, 993-1004 (1982).

6. Ели с е е в П. Г., Препринт ФИАН, № 33, 1970.

7. B o te z, D., IEEE J. Quant. Electron., 14, 230-232 (1978).

Ннститут физики

Академии наук Эстонской ССР
Поступила в редакцию 21/IV 1982 